\title{
Genetic and functional characterization of the NanA sialidase from Clostridium chauvoei
}

Edy M Vilei ${ }^{i^{* \dagger}}$, Anders Johansson ${ }^{1,2 \dagger}$, Yvonne Schlatter $^{1}$, Keith Redhead ${ }^{3}$, Joachim Frey ${ }^{1}$

\begin{abstract}
Clostridium chauvoei is the causative agent of blackleg, a wide spread serious infection of cattle and sheep with high mortality. In this study we have analyzed the sialidase activity of the NanA protein of C. chauvoei and cloned the sialidase gene nanA. Sialidase is encoded as a precursor protein of 722 amino acids with a 26 amino acid signal peptide. The mature sialidase has a calculated molecular mass of $81 \mathrm{kDa}$ and contains the carbohydrate binding module 32 (CBM32, or F5/8 type C domain), the sialic acid binding module CBM40 and the enzymatically active sialidase domain found in all pro- and eukaryotic sialidases. Sialidase activity does not require the CBM32 domain. The NanA protein is secreted by $C$. chauvoei as a dimer. The nanA gene was found to be conserved and sialidase activity was found in C. chauvoei strains isolated over a period of 50 years from various geographical locations. Antiserum directed against a recombinant $40 \mathrm{kDa}$ peptide containing CBM40 and part of the enzymatically active domain of NanA neutralized the secreted sialidase activity of all $C$. chauvoei strains tested.
\end{abstract}

\section{Introduction}

Clostridium chauvoei is an anaerobic, endospore-forming Gram-positive bacterium known to be the causative agent of blackleg, a severe disease with high mortality affecting young cattle and sheep of any age. While $C$. chauvoei infections are specifically found in ruminants, a case of human fulminant gas gangrene caused by $C$. chauvoei was recently described [1]. Ingestion of $C$. chauvoei spores is probably the most common form of exposure and infected ruminants do not directly transmit the disease to other animals. The endospores of $C$. chauvoei can lie dormant in the soil for years and, after ingestion by the animal, they are assumed to cross over the gastro-intestinal tract, enter the bloodstream and finally migrate in various organs and muscles, where they remain dormant until stimulated to cause disease [2]. These spores are only activated in a low oxygen environment, such as that of damaged or bruised tissue in cattle. In sheep, blackleg is frequently associated with wounding, such as at shearing cuts, tail docking and castration site [3]. Once these stimulating events occur, the spores in the infected tissue germinate and multiply into

\footnotetext{
* Correspondence: edy.vilei@vbi.unibe.ch

+ Contributed equally

'Institute of Veterinary Bacteriology, Vetsuisse Faculty, University of Bern, Bern, Switzerland

Full list of author information is available at the end of the article
}

the disease-causing C. chauvoei bacteria. Then, the disease progresses rapidly, with the infected animal dying in 12 to $36 \mathrm{~h}$ after the appearance of the first symptoms [4]. The molecular mechanisms of blackleg are not clearly understood. Due to the peracute evolution with severe oedematous lesions restricted to the local area, fever, loss of appetite, lameness and depression observed in blackleg outbreaks, it is speculated that virulence of C. chauvoei is caused by rapid spread of the activated, vegetative form of the bacterium in the infected tissue, followed by the production of potent toxins $[4,5]$. Although sialidases and toxins produced by C. chauvoei are believed to play a significant role in the spread of the pathogen and in the induction of lesions in affected tissues [5], they have not yet been characterized at the molecular and genetic level. It has to be mentioned here that, to date, only a few DNA sequences of $C$. chauvoei have been deposited in DDBJ/EMBL/GenBank databases including the $16 \mathrm{~S}$ and the $23 \mathrm{~S}$ rRNA genes, the 16S-23S rRNA spacer region, and the flagellin gene $[6,7]$.

Sialidases, or neuraminidases (EC 3.2.1.18), are enzymes that cleave $\mathrm{N}$-acetylneuraminic acid from carbohydrate polymers, such as mucin, glycoproteins, gangliosides and other sialoglycoconjugates, located on many mammalian cell membranes. The mature sialidase is generally composed of three main domains (Figure 1). At its $\mathrm{N}$-terminal end, it contains the F5/8 type $\mathrm{C}$ 


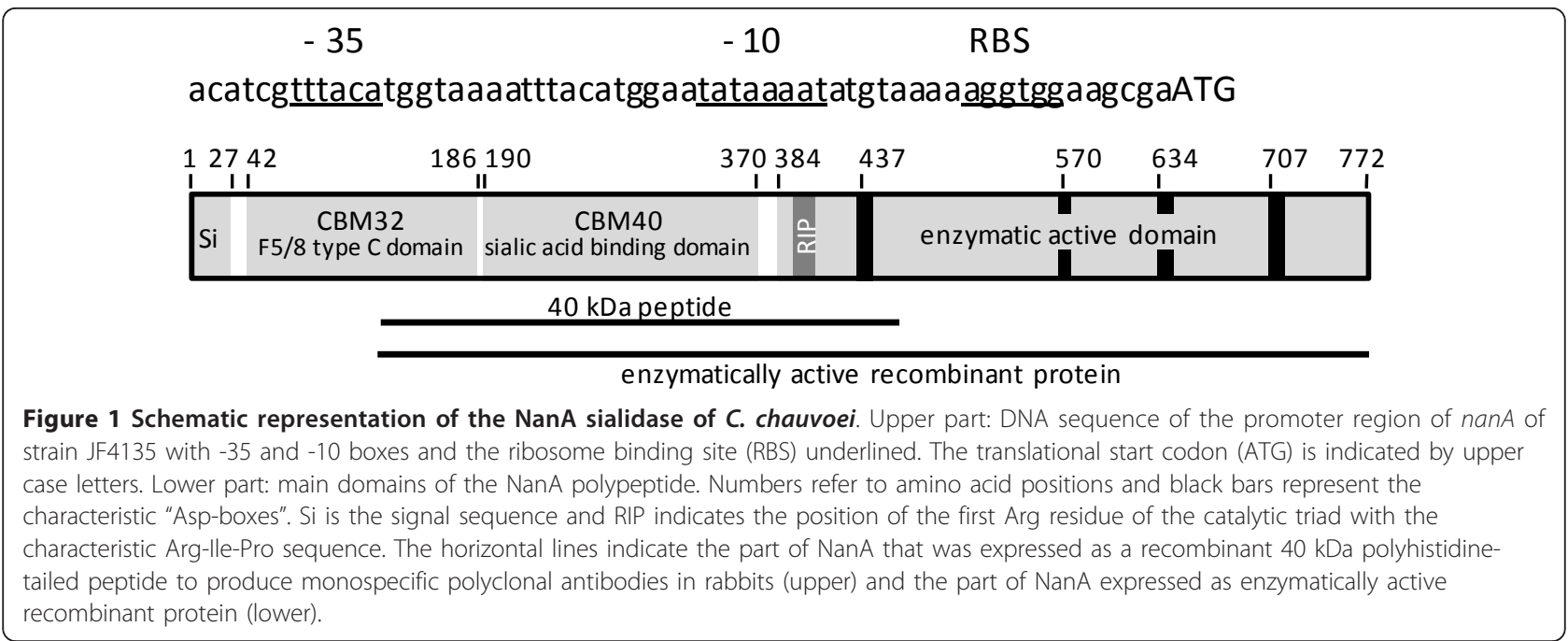

domain, alternatively designated CBM32 (carbohydrate binding module 32) or discoidin domain, that appears to be primarily involved in binding of terminal galactoconfigured sugar residues and is also found in the $C$. perfringens sialidase NanJ [8]. The central domain contains a CBM40 module (or lectin-like sialic acid binding domain) that was shown to be specific for binding sialic acid residues in NanJ of $C$. perfringens [8]. The enzymatically active site of sialidases is in the C-terminal end that contains the usual catalytic residues that are common to all sialidases [9-11], including a catalytic nucleophile tyrosine, an arginine triad that interacts with the carboxylate group of sialic acid, a tryptophan residue that forms a weak $\mathrm{H}$-bond with the glycerol side chain of sialic acid, and an aspartic acid that forms $\mathrm{H}$-bonds with the four $\mathrm{OH}$-groups of sialic acid. Furthermore, the enzymatically active domain contains four "Asp-boxes" [10] characterized by the motif Ser-X-Asp-X-Gly-XThr-Trp, and the first arginine of the catalytic triad of sialidases generally belongs to a RIP (Arg-Ile-Pro) motif [12].

Sialidases have been found in viruses, bacteria, protozoa, fungi and metazoans [13]. In viruses, parasites and bacteria, sialidases are thought to be important for survival and pathogenicity of the organisms [14]. It has been established that viral sialidases have a central role in the pathogenesis of influenza $[14,15]$. The importance of sialidase in respiratory tract infections has been demonstrated for two bacterial species: Streptococcus pneumoniae and Pseudomonas aeruginosa. The neuraminidases (sialidases) NanA and NanB of S. pneumoniae have been shown to be essential for successful colonization of both upper and lower respiratory tract and for pneumococcal survival in the blood using an outbred MF1 mouse or chinchilla model $[16,17]$. The sialidase produced by $P$. aeruginosa has been shown to be a critical factor for colonization of the upper respiratory tract. It has also been shown that viral neuraminidase inhibitors blocked biofilm production and the bacterial colonization by P. aeruginosa [18]. Recently, Mally and colleagues [19] demonstrated the impact of sialidase in Capnocytophaga canimorsus, a commensal canine bacterium of the oral flora, that can cause severe septicaemia and meningitis in humans [20]. Wild type $C$. canimorsus is dependent on a surface exposed sialidase for growth and persistence in a murine infection model, whereas a sialidase deficient $C$. canimorsus mutant was unable to grow and persist in mice [19]. Sialidase may have an important role in blackleg by degrading tight junctions upon cleavage of sialic acids at a high rate, thus allowing the bacterium to spread through host tissue [5]. A sialidase activity has been reported in $C$. chauvoei [21], but the sialidase enzyme has not yet been characterized in detail [22,23]. Furthermore, the molecular and genetic basis of sialidase in C. chauvoei is still unclear.

This study reports the cloning of the nanA sialidase gene from $C$. chauvoei, its expression and the characterization of the sialidase NanA protein. Under native conditions in bacterial culture medium, C. chauvoei sialidase is found as a $150 \mathrm{kDa}$ protein, in the form of a dimer of two NanA peptides with an apparent molecular mass of $72 \mathrm{kDa}$.

\section{Materials and methods}

Strains and culture conditions, DNA extraction and sequence analysis

The seven $C$. chauvoei strains used in this study are listed in Table 1 . The $C$. chauvoei strains were grown anaerobically on Tryptic Soy Agar (TSA) medium containing 5\% sheep erythrocytes (bioMérieux, Geneva, Switzerland) or in TGY broth (3\% tryptic soy broth 
Table 1 Clostridium chauvoei strains used in this study

\begin{tabular}{lll}
\hline $\begin{array}{l}\text { Internal } \\
\text { reference }\end{array}$ & Strain & Description and origin \\
\hline JF1863 & ATCC10092 & $\begin{array}{l}\text { type strain } \\
\text { blackleg outbreak, Brazil, 2002 } \\
\text { JF2697 }\end{array}$ \\
MT & acute blackleg, New Zealand, \\
JF3703 & C6 O59 & acute blackleg, Switzerland, 2004 \\
JF4135 & 04 & blackleg outbreak, Sweden, 2002 \\
JF4251 & AN2548/02 & blackleg outbreak, Sweden, 2006 \\
JF4252 & AN1717/06 & blackleg outbreak, Sweden, 2007 \\
JF4253 & AN2500/07 &
\end{tabular}

(Becton Dickinson AG, Allschwil, Switzerland), 2\% glucose (Sigma-Aldrich chemical, St. Louis, MO, USA), 1\% yeast extract (Becton Dickinson AG), and $0.1 \% \mathrm{~L}$ cysteine (Sigma-Aldrich chemical)) at $37^{\circ} \mathrm{C}$ for up to 72 h. Escherichia coli strains were grown in Luria-Bertani (LB) broth or on LB agar at $37^{\circ} \mathrm{C}$. Antibiotics (ampicillin, $100 \mu \mathrm{g} / \mathrm{mL}$ ) were added for selection of $E$. coli carrying recombinant plasmids.

DNA from C. chauvoei strains was extracted by the guanidium thiocyanate method [24]. Ligation, subcloning, plasmid extraction of the DNA fragments and agarose gel electrophoresis $(0.7 \%)$ were performed as described [25]. Plasmid extraction from recombinant $E$. coli clones was done by alkaline lysis using a Miniprep kit (PeqLab Biotechnologie GmbH, Erlangen, Germany). Sequence analysis was performed using an ampli-Taq FS dye terminator kit (Applied Biosystems, Foster City, CA, USA) and the universal primers complementary to the $\mathrm{T} 3$ and T7 promoters flanking the multiple cloning site of $\mathrm{pBK}-\mathrm{CMV}$, or primers that were derived from obtained DNA sequences.

Comparisons of DNA sequences and their corresponding amino acid (aa) sequences with DDBJ/EMBL/GenBank databases were performed using BLAST analysis [26]. The molecular mass and theoretical isoelectric points (pI) of the sialidase were calculated using ProtParam [27]. Pattern and profile searches were carried out at the online servers Motif Scan [28] and InterProScan [29]. The presence and location of signal peptide cleavage sites in the amino acid sequence were predicted with the program SignalP 3.0 [30]. Prediction of transmembrane helices and topology of the sialidase was carried out with the programs HMMTOP 2.0 [31] and Phobius [32]. The neighbour joining tree of aa sequences from clostridial sialidases was constructed with multiple amino acid sequence alignment by ClustalW2 [33].

\section{Production of rabbit serum directed against proteins secreted by $C$. chauvoei}

A culture $(100 \mathrm{~mL})$ of C. chauvoei strain JF1863 (Table 1; type strain ATCC10092) grown for $72 \mathrm{~h}$ in TGY medium was centrifuged at $9000 \times g$ at $4^{\circ} \mathrm{C}$ for $20 \mathrm{~min}$. Proteins in the supernatant were precipitated overnight at $4^{\circ} \mathrm{C}$ upon addition of $70 \%(\mathrm{w} / \mathrm{v})$ ammonium sulphate. Precipitated proteins were sedimented by centrifugation for $10 \mathrm{~min}$ at $12000 \times \mathrm{g}$. The pellet was suspended in 10 $\mathrm{mL}$ of dialysis buffer (0.1 M Na-bicarbonate, $\mathrm{pH} 8.3$, $0.25 \mathrm{M} \mathrm{NaCl}$ ) and then dialyzed overnight at $4^{\circ} \mathrm{C}$ with gentle stirring. Aliquots of $500 \mu \mathrm{L}$ containing $200 \mu \mathrm{g}$ of dialyzed proteins or $50 \mu \mathrm{g}$ of purified recombinant protein were mixed with the same quantity of Gerbu adjuvant (Gerbu Biotechnik GmbH, Gaiberg, Germany) for immunizing rabbits three times at intervals of two weeks. Rabbits were bled two weeks after the last immunization and all serum samples were then incubated for $30 \mathrm{~min}$ at $56^{\circ} \mathrm{C}$ to inactivate complement.

\section{Construction of a genomic library, cloning of the nanA gene and DNA sequence analysis}

Genomic DNA of C. chauvoei strain JF4135 (other name: C6 O/D1126/04; Table 1), a strain that caused acute blackleg in Switzerland in 2004, was partially digested with Sau3AI and fragments from 2 to $4 \mathrm{~kb}$ were selected to construct a genomic library, using BamHI-digested $\lambda$-ZAP-express vector arms, which was packaged with the Gigapack-11 packaging system (Stratagene, La Jolla, CA, USA). The phage library was plated using standard protocols on the E. coli strain XL-1 Blue MRF'. Screening of transformed $E$. coli was performed with rabbit serum against secreted proteins of $C$. chauvoei, raised as described above, used at a dilution of 1:100. Positive clones were amplified and finally subjected to in vivo excision using the $\mathrm{f} 1$ helper phage in $E$. coli strain XLOLR to obtain phagemid clones in expression vector $\mathrm{pBK}-\mathrm{CMV}$. A clone that strongly reacted with the rabbit hyper-immune serum was revealed to contain a $1.7 \mathrm{~kb}$ fragment whose sequence showed similarity to the sialidase gene of Clostridium septicum [34], as determined by BLAST analysis. The remaining part of the sialidase gene, named nanA (neuraminidase A), was detected by screening the same genomic DNA library by DNA:DNA hybridization with a 512 bp digoxigenin-labelled probe amplified from the cloned $1.7 \mathrm{kDa}$ fragment with primers digSiaCC_605F (TTACTAGTGGAAATGGTG) and digSiaCC_1117R (CTTTTGCTGTAGTTTCAC). Digoxigenin labelling of the probe was done according to the manufacturer's instructions (Roche Diagnostics, Rotkreuz, Switzerland). Upon analysis of few clones harbouring partial sequences of nanA by primer walking with an $A B I$ Prism model 3100 genetic analyzer (Applied Biosystems), the complete DNA sequence of the cloned gene and the sequences upstream and downstream were determined. Sequence alignment and editing were performed using the software Sequencher 3.0 (GeneCodes, 
Ann Arbor, MI, USA). The full length nanA gene of strain JF3703 (other name: 595E; Table 1), a highly virulent strain isolated in New Zealand in 1959, was determined by PCR amplification and DNA sequence analysis using the same primers as for strain JF4135. The sequence data of the sialidase gene $\operatorname{nan} A$ have been deposited in the EMBL Nucleotide Sequence Database (European Bioinformatics Institute, Cambridge, UK) under accession numbers FM213081 for strain JF3703 and FM213082 for strain JF4135.

\section{PCR analysis}

The presence of the nanA gene of $C$. chauvoei was demonstrated in all seven $C$. chauvoei strains examined (Table 1) by PCR using the primers SiaCC_1371F (ATCAGCAATAGATACATC) and SiaCC_1789R (TGACCTCTTCCTGGTCCTGT). PCR was carried out in $30 \mu \mathrm{L}$ reaction mixtures containing $1 \times$ reaction buffer B (supplied with FIREPol ${ }^{\circledR}$ DNA polymerase), 2.5 $\mathrm{mM} \mathrm{MgCl} 2,0.4 \mathrm{mM}$ of each primer, $1 \mathrm{mM}$ dNTPs, and $2.5 \mathrm{U}$ of FIREPol ${ }^{\circledR}$ polymerase (Solis BioDyne, Tartu, Estonia). Approximately $100 \mathrm{ng}$ of genomic DNA was added as template. Cycling conditions on a 9800 Fast Thermal Cycler (Applied Biosystems) were 3 min denaturation at $94^{\circ} \mathrm{C}$, followed by 35 cycles at $94^{\circ} \mathrm{C}$ for $30 \mathrm{~s}$, $56^{\circ} \mathrm{C}$ for $30 \mathrm{~s}$ and $72^{\circ} \mathrm{C}$ for $60 \mathrm{~s}$. A final extension step for $7 \mathrm{~min}$ at $72^{\circ} \mathrm{C}$ was included. Amplicons were purified with the High pure PCR product purification kit (Roche Diagnostics).

\section{Cloning and expression of the CBM40 module of the $C$. chauvoei sialidase and production of antibodies}

To generate polyclonal antibodies directed against the CBM40 sialic acid binding domain of NanA, the coding region from nt position 367 to 1389 of the nanA gene (corresponding to aa 123 to 463), which also includes the first Arg residue and the RIP (Arg-Ile-Pro) motif of the catalytic site of sialidases, was amplified from $C$. chauvoei strain JF4135 using the primers Sia40_NcoIF (ggtcccatggGTATAAAAGAATACAAAATTTATGCG) and Sia40_HindIIIR (cccaagcttGATGTATCTATTGCTGATGATGCTCC) (nucleotides in lower case are extensions and underlined nucleotides are recognition sites for the restriction enzymes NcoI or HindIII). The resulting PCR product was cloned into the pGEM-T easy vector (Promega Corp., Madison, WI, USA) and transformed into E. coli strain DH5a. Recombinant plasmids with the correct sequence were then digested with NcoI and HindIII, and the DNA fragments were inserted into the expression vector pETHIS-1 [35]. The cloned insert was sequenced to ensure correct fusion with the vector's poly-His codons before being transformed into E. coli BL21(DE3) cells [36] for expression. Induction and subsequent purification of the recombinant $40 \mathrm{kDa}$
His-tagged protein was performed as described [37]. Purified $40 \mathrm{kDa}$ His-tagged protein was used to immunize rabbits as described above.

\section{SDS-PAGE and immunoblotting}

Pellets and supernatants of C. chauvoei strains (Table 1) incubated at $37^{\circ} \mathrm{C}$ under anaerobic conditions in TGY medium for $72 \mathrm{~h}$ were examined by sodium dodecyl sulfate-polyacrylamide gel electrophoresis (SDS-PAGE) and immunoblotting on nitrocellulose membranes (Bio-Rad Laboratories, Hercules, CA, USA). Proteins were separated on $12 \%$ acrylamide gels by SDS-PAGE under denaturing or non-denaturing conditions [38]. Nondenaturing gel electrophoresis was performed in the absence of reducing agents. In order to detect the sialidase, the immunoblot membranes were incubated with rabbit polyclonal anti-sialidase $40 \mathrm{kDa}$ peptide serum (diluted 1:1000), followed by incubation with a phosphate- or peroxidase-conjugated goat anti-rabbit IgG heavy and light chains (Kirkegaard \& Perry Laboratories, Gaithersburg, MD, USA) diluted 1:2000.

\section{Expression of functional NanA in E. coli DH5 $\alpha$}

To express enzymatically active recombinant NanA, we amplified the part of the nanA gene from nt position 364 to 2316 (corresponding to aa 122 to 772 ), encoding CBM40 (sialic acid binding module) and the enzymatically active domain but not CBM32 (Figure 1), from C. chauvoei strain JF4135 using the primers SiaCC_BssHIIF (ttggcgcgcAATGATAAAAGAATACAAAATTTATGCG) and SiaCC_HindIIIR (cccaagcttTATAAATTTCCATTTTCTGTTATTAAACC) (nucleotides in lower case are extensions containing recognition sites for the restriction enzymes BssHII and HindIII, respectively, that are underlined). The resulting PCR product was cloned into the vector T-easy (Promega Corp.) and transformed into $E$. coli strain DH5 $\alpha$. Recombinant plasmids with the correct sequence were then digested with Bss HII and HindIII, and the DNA fragment was ligated into the multiple cloning site of vector pFastBac1 (Invitrogen, Carlsbad, CA, USA) digested with BssHII and HindIII. Vector pFastBac1 was chosen in order to express active NanA in both prokaryotic and eukaryotic cells, such as insect cells, utilizing the baculovirus expression system. This plasmid harbours the polyhedrin promoter from the baculovirus, which was shown to also have an adequate ability for initiating expression of introduced heterologous genes in E. coli [39]. E. coli DH5a was transformed with pFastBac1::nanA and with empty vector pFastBac1. Sialidase activity of recombinant $E$. coli was measured using the sialidase spot test described below.

\section{Sialidase activity tests}

The fluorogenic substrate 2'-(4-methylumbelliferyl)- $\alpha-\mathrm{d}$ $\mathrm{N}$-acetylneuraminic acid (MU-Neu5Ac) (Sigma-Aldrich 
chemical) was used to assay sialidase activity in a spot test [40] and in a fluorimetric determination in culture fluids $[41,42]$.

For the spot test, a working solution of $15 \mu \mathrm{M}$ MUNeu5Ac in $0.17 \mathrm{M}$ sodium acetate buffer ( $\mathrm{pH}$ 6.5) was prepared. Either colonies of $C$. chauvoei, recombinant $E$. coli, or $10 \mu \mathrm{L}$ of supernatant from liquid C. chauvoei cultures were spotted onto 1-mm Whatman filter paper that had been previously moistened with the MUNeu5Ac working solution, and then incubated at $37^{\circ} \mathrm{C}$ for $15 \mathrm{~min}$. The reactions were stopped $(0.085 \mathrm{M}$ glycine, $0.2 \mathrm{M}$ sodium carbonate, $\mathrm{pH} 9.4$ ), the filter papers air-dried and fluorescence observed under a UV light (360 $\mathrm{nm}$ excitation wavelength). Observable bright blue fluorescence was recorded as positive. Anti-sialidase 40 $\mathrm{kDa}$ peptide serum or pre-immune serum (both diluted 1:10) obtained from the same rabbit were spotted onto the filter paper, at some distance from the bacterial spots but still partially overlying them, to assess the neutralizing effect of the anti-sialidase serum against sialidase activity.

For the fluorimetric determination of culture fluid sialidase activity, strain JF4135 was grown anaerobically for 1 day at $37^{\circ} \mathrm{C}$. After cell counting $\left(\mathrm{OD}_{600 \mathrm{~nm}}\right)$, the culture was centrifuged at $2000 \times g$ for $10 \mathrm{~min}$ and the supernatant was centrifuged again at $15000 \times g$ for 15 min to obtain cell-free supernatant. The reaction was performed in a total volume of $100 \mu \mathrm{L}$ containing $30 \mu \mathrm{L}$ of $0.1 \mathrm{M}$ sodium acetate buffer ( $\mathrm{pH} 6.0), 10 \mu \mathrm{L}$ of a 1 $\mathrm{mM}$ MU-Neu5Ac solution in water and $60 \mu \mathrm{L}$ of sample (each sample consisted of $54 \mu \mathrm{L}$ cell-free supernatant pre-incubated for $30 \mathrm{~min}$ at $37^{\circ} \mathrm{C}$ with $6 \mu \mathrm{L}$ of anti-sialidase or pre-immune serum (at several dilutions) prior to fluorimetric quantification of sialidase activity). Samples were incubated at $37^{\circ} \mathrm{C}$ for $60 \mathrm{~min}$ and quantification of sialidase activity was carried out kinetically (at $30 \mathrm{sec}$ intervals) in triplicate with an FLx800 fluorescence microplate reader (BioTek, Luzern, Switzerland). Liberated 4-methylumbelliferone (MU) was quantified at 360/ $40 \mathrm{~nm}$ excitation and $460 / 40 \mathrm{~nm}$ emission in relation to a calibration curve obtained with MU standards (serial 2 -fold dilutions ranging from 0 to $100 \mu \mathrm{M}$ ).

\section{Results}

\section{Characteristics of the Clostridium chauvoei sialidase}

The full length sialidase gene nanA of $C$. chauvoei strain JF4135 consists of $2319 \mathrm{bp}$. A strong ribosome binding site (AGGTGG) is found eight bases upstream of the putative start codon ATG and is preceded by a potential clostridial promoter sequence including a TATA box (TATAAAAT) and a -35 element (TTTACA) (Figure 1). The NanA protein derived from the nucleotide sequence consists of 772 aa, with a predicted molecular mass of $84.0 \mathrm{kDa}$ and a pI of 4.89. The aa sequence derived from the DNA sequence reveals a leader sequence peptidase cleavage site between position 26 and 27 (Ile-TyrAla $\downarrow$ Asp-Ile), which is characteristic for secreted proteins of Gram-positive bacteria. The putative mature sialidase consists of 746 aa with a calculated molecular mass of $81.1 \mathrm{kDa}$. At its $\mathrm{N}$-terminal end, aa residues 42 to 186 (numbering always includes the aa residues of the precursor) form the CBM32 module (Figure 1). In the central portion of NanA, aa 190 to 370 form the CBM40 module. The enzymatically active site ranging from aa 384 to 770 contains the usual catalytic residues, common to all sialidases: Tyr-728 (catalytic nucleophile), Arg-400, Arg-613 and Arg-693 (forming the arginine triad, whereas Arg-400 is contained in the RIP motif), Trp-577 (that interacts with the glycerol side chain of sialic acid) and Asp-582 (that forms H-bonds with the four $\mathrm{OH}$-groups of sialic acid). Moreover, the four "Aspboxes" are found at aa positions 437-444, 570-577, 634641 and 707-714 (Figure 1).

The aa sequence of NanA from strain JF3703 differs from that of JF4135 at 18 aa loci that do not affect catalytic domains or "Asp-boxes". Amino acid similarities to NanA were found with several clostridial sialidases (Figure 2). Most closely related is the sialidase from C. septicum with an aa homology of $79 \%$ and $82 \%$ identical nucleotides at the gene level.

\section{Sialidase activity and presence of nanA in C. chauvoei strains}

All seven C. chauvoei strains used (Table 1) contained the nanA gene as revealed by PCR. Sequencing of the amplicons revealed minor nucleotide changes that do not affect however the size of expressed proteins. Monospecific polyclonal antibodies directed against the recombinant $40 \mathrm{kDa}$ peptide representing the CBM40 domain of NanA (Figure 1) reacted on standard immunoblots containing supernatants of $C$. chauvoei cultures of all strains with a $150 \mathrm{kDa}$ and to a lesser extent with a $72 \mathrm{kDa}$ band (Figure 3 shows the results for strains JF3703 and JF4135). The latter band was somewhat smaller than the predicted molecular mass of $81 \mathrm{kDa}$ for mature NanA calculated from the DNA sequence of the $n a n A$ gene. When non-denaturing gels were used for the immunoblots, only the $150 \mathrm{kDa}$ band was detected (Figure 3), indicating that the C. chauvoei sialidase is a $150 \mathrm{kDa}$ dimer of the $72 \mathrm{kDa}$ NanA polypeptide.

All strains were positive in the sialidase spot test, showing the same spot diameter. Sialidase activity was observed directly with bacterial colonies spotted onto the filter, as well as with supernatant of C. chauvoei cultures grown in TGY medium (Figure 4), showing that sialidase was secreted. Moreover, when part of the nanA gene encoding the lectin-like sialic acid binding module CBM40 and the enzymatically active domain, but not 

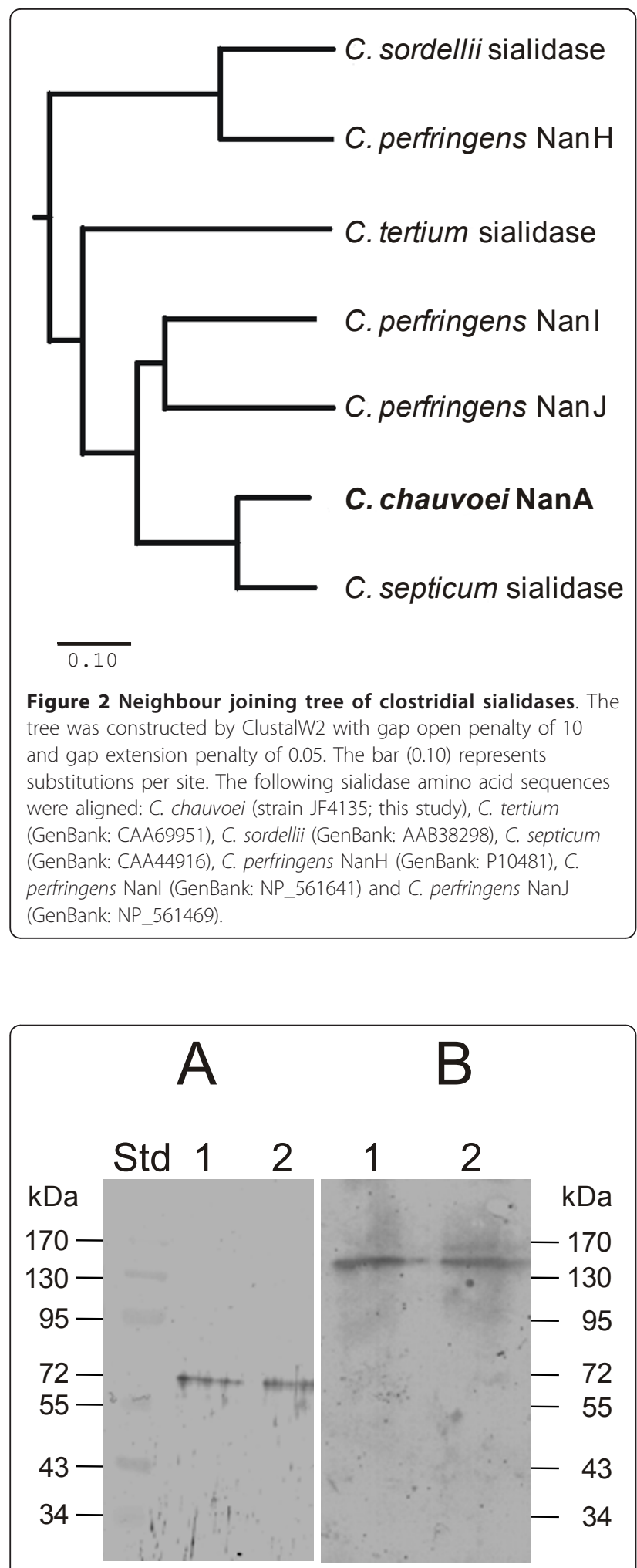

Figure 3 Dimer form of NanA. Western blot with anti-sialidase 40 $\mathrm{kDa}$ peptide serum under denaturing conditions (panel A) showing the $72 \mathrm{kDa}$ monomer, or under native conditions (panel B) showing the $150 \mathrm{kDa}$ dimer. Lane 1, C. chauvoei strain JF3703; lane 2, C. chauvoei strain JF4135.

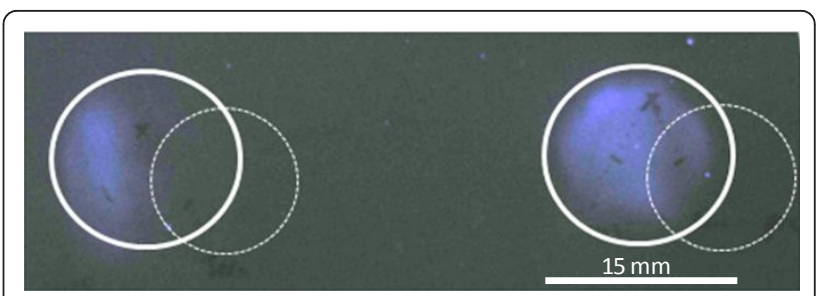

Figure 4 Sialidase neutralization spot test. The solid rings show the diffusion zone of the culture supernatant of $C$. chauvoei strain JF4135. The dotted rings show the diffusion zone of: left, antisialidase $40 \mathrm{kDa}$ peptide serum; right, pre-immune serum from the same rabbit.

CBM32, was cloned into plasmid pFastBac1 and transformed into $E$. coli strain DH5 $\alpha$, sialidase activity was found in culture supernatant of the recombinant $E$. coli strain harbouring the plasmid pFastbac1::nanA but not in the $E$. coli control strain harbouring the empty vector pFastBac1 only (not shown). Sialidase activity secreted by all C. chauvoei strains used in this study and by recombinant $E$. coli was neutralized by monospecific polyclonal antiserum raised in rabbit against the recombinant $40 \mathrm{kDa}$ peptide (Figure 4 shows the results for strain JF4135), showing the functional impact of the CBM40 module covered by this peptide.

Similarly, the anti-sialidase $40 \mathrm{kDa}$ peptide serum had an inhibitory effect on the sialidase activity in culture supernatants of $C$. chauvoei as determined by a kinetic fluorimetric assay (Figure 5 shows the results for strain JF4135). The assay monitored the cleavage of the glycosidic linkage of $100 \mu \mathrm{M}$ of substrate MU-Neu5Ac upon incubation at $37^{\circ} \mathrm{C}$ for $60 \mathrm{~min}$ with C. chauvoei culture fluids. While the culture fluid of $5 \times 10^{7}$ cells was able to release approximately $10 \mu \mathrm{M} \mathrm{MU}$ from the substrate, anti-sialidase serum could inhibit this activity by $60 \%$ when diluted 1:10 (Figure 5). Anti-sialidase serum diluted 1:100 and 1:1000 inhibited the activity by $40 \%$ and $20 \%$, respectively. As expected, pre-immune serum from the same rabbit, used as a control at different dilutions, did not affect the C. chauvoei culture fluid sialidase activity.

\section{Discussion}

Due to the rapid progression and severity of blackleg, it is appropriate to assume that potent toxins are responsible for the rapid spread of C. chauvoei and for the severe lesions that are produced by the pathogen in the target tissues. It has previously been hypothesized that sialidase may have an important role in blackleg by aiding the spread of the bacterium through the tissue [5]. Furthermore, sialidases from S. pneumoniae, $P$. aeruginosa and $C$. canimorsus have been demonstrated to be crucial for infection and colonization of the host [16-19]. From these data, it can be hypothesized that 


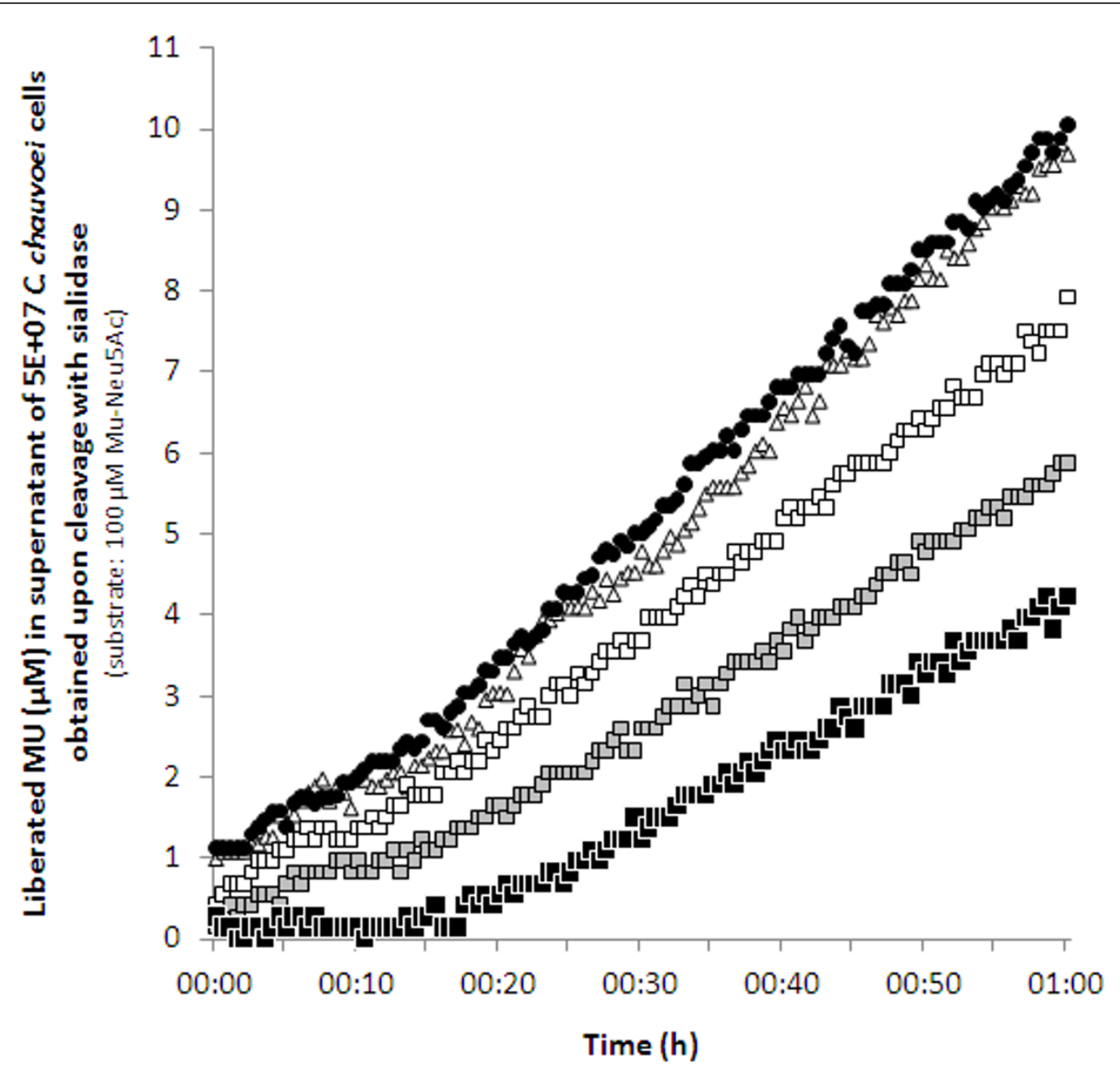

Figure 5 Fluorimetric determination of culture fluid sialidase activity. Cell-free supernatant of C. chauvoei strain JF4135 was pre-incubated with different dilutions of anti-sialidase $40 \mathrm{kDa}$ peptide serum or pre-immune serum from the same rabbit. Liberated 4-methylumbelliferone (MU) obtained upon cleavage of substrate MU-Neu5Ac with sialidase in culture fluids of $5 \times 10^{7} \mathrm{C}$. chauvoei cells was recorded at $30 \mathrm{sec}$ intervals. Black circles, no pre-incubation with antibodies; white triangles, pre-incubation with pre-immune serum (diluted 1:10); squares, preincubation with anti-sialidase serum (white, diluted 1:1000; gray, diluted 1:100; black, diluted 1:10). Plots are the mean of three kinetic measurements of sialidase activity.

the sialidase of $C$. chauvoei might have an important role in the early stage of blackleg infection by degrading tight junctions upon cleavage of sialic acid from mucins and glycoproteins in the infected tissue. Cleavage of sialic acid results in decreased rigidity of the cell surface, thereby facilitating cell motility and thus rendering the target site vulnerable to massive attack by the pathogen that rapidly spreads in the infected tissue. Then, the production of toxins such as hyaluronidases (gammatoxins), deoxyribonucleases (beta-toxins) and oxygenlabile hemolysins by these bacteria may give rise to necrosis of the infected muscles and alteration of vascular endothelium, both known as characteristic signs of blackleg [5].
Sialidase activity of C. chauvoei was reported previously [21] but the corresponding enzyme was characterized only biochemically and not genetically [22,23]. Our genetic and biochemical data reveal a sialidase, named NanA, in C. chauvoei that is a $150 \mathrm{kDa}$ homodimer composed of two identical polypeptides of an apparent molecular mass of $72 \mathrm{kDa}$. Differences between the predicted size calculated from the nucleotide sequence of $n a n A(81 \mathrm{kDa})$ and the apparent size observed by SDS-PAGE (72 kDa) have previously been reported for several bacterial sialidases, such as $S$. pneumoniae [43,44], Clostridium tertium [45] and C. septicum [46]. Native sialidase enzymes from bacteria are described as multimers, mostly dimers, of polypeptides 
having molecular masses ranging between 50 and 80 $\mathrm{kDa}$ [45], which agrees with our data for the sialidase of C. chauvoei. Although the $300 \mathrm{kDa}$ sialidase described by Heuermann et al. [22] might represent a multimer of the $72 \mathrm{kDa}$ NanA protein, we did not detect such a protein on our immunoblots. The sialidase NanA of $C$. chauvoei is most closely related to the sialidase of $C$. septicum and to NanJ and NanI of C. perfringens (Figure $2)$, the structures of which have been studied in detail $[8,10]$. In this respect, it is interesting to note that $C$. chauvoei and C. septicum are phylogenetically very closely related [47] and at one time were classified phenotypically as the same species [48].

The C. chauvoei sialidase NanA displays the same motifs as the $C$. perfringens sialidase NanJ [10]. NanA has a CBM32, or F5/8 type $\mathrm{C}$ domain, located at position 42 to 186 , which is absent in C. perfringens NanI $[8,10]$. This domain does not seem to be essential for enzyme activity as demonstrated by the sialidase activity of the recombinant NanA without CBM32. This is in agreement with recent data that classify the CBM32 module as a special domain that is involved in binding complex sialic acid substrates containing galactose residues $[9,11]$. The lectin-like sialic acid binding module CBM40 and the enzymatic active domains show strong similarities to NanI and NanJ of $C$. perfringens. The latter domain contains all the characteristic residues necessary for sialidase activity, as seen in all prokaryotic and eukaryotic sialidases [11], including the catalytic arginine triad with the RIP motif on the first of the three Arg residues, and the characteristic "Asp-boxes". Antibodies directed against the recombinant $40 \mathrm{kDa}$ peptide that includes the entire CBM40 module and part of the enzymatically active domain of NanA, in particular the RIP motif (Figure 1), neutralized the secreted sialidase activity of C. chauvoei strains (Figures 4 and 5), confirming their importance in sialidase activity. Furthermore, this result suggests that NanA is the sole or predominant secreted sialidase of $C$. chauvoei. If other sialidases antigenically similar to NanA were present in C. chauvoei, supplementary protein bands would have been detected on immunoblots using the same antiserum against the $40 \mathrm{kDa}$ peptide, but no such additional sialidases were observed (Figure 3).

Since prevention of contact of ruminants with the endospores of C. chauvoei that are present in the contaminated soil is virtually impossible, vaccination is the only preventive measure to effectively control blackleg. Current vaccination strategy against this disease relies on the use of a suspension for injection containing formaldehyde-inactivated cultures of virulent strains of $C$. chauvoei and culture supernatant, generally presented in polyvalent formulations. These vaccines are efficient but the particular antigens necessary to induce protective immunity against blackleg are unknown, thus requiring expensive work-intensive animal models for potency testing of vaccine batches. Moreover, molecular quality control of current vaccines, design of novel vaccines and diagnostic tests are mainly hampered by the lack of basic knowledge on the molecular mechanisms of pathogenicity of C. chauvoei. Studies on the role of sialidases of Streptococcus pneumoniae in otitis media in children and of Propionibacterium acnes in acne vulgaris have shown that sialidases of these species induce antibodies that inhibit propagation of bacteria in the host tissues $[17,49,50]$. When challenged, chinchillas vaccinated with recombinant sialidase from S. pneumoniae, expressed in E. coli, showed significantly reduced colonization and middle ear invasion compared with non-vaccinated individuals. Taken together, these findings in animal studies and the data of the present work, whereby antibodies raised against the sialidase $40 \mathrm{kDa}$ peptide of $C$. chauvoei effectively neutralized the activity of the infectious agent, indicate that sialidase of $C$. chauvoei may be a good vaccine candidate to protect ruminants against the pathogen and to prevent the occurrence of blackleg. Furthermore, we expect that a combination of this antigen with one or more polypeptides derived from the toxins produced by $C$. chauvoei may have a potentiated protective effect against blackleg.

\section{Acknowledgements}

This study was financially supported by a grant from Intervet/ScheringPlough Animal Health. The authors are grateful to Elisabeth Bagge (National Veterinary Institute, Uppsala, Sweden) for kindly providing isolates from Swedish outbreaks of blackleg.

\section{Author details}

${ }^{1}$ Institute of Veterinary Bacteriology, Vetsuisse Faculty, University of Bern, Bern, Switzerland. ${ }^{2}$ National Food Administration, Uppsala, Sweden. ${ }^{3}$ Intervet/ Schering-Plough Animal Health, Milton Keynes, UK.

\section{Authors' contributions}

EMV and AJ made equivalent contributions to the study. EMV carried out the fluorimetric determination of culture fluid sialidase activity, devised the cloning strategies and participated in the sequence analysis. He drafted the manuscript. AJ constructed and screened the genomic library. He carried out the molecular biological studies and was responsible for expressing the recombinant sialidases as well as testing the resulting polypeptides in immunoblotting and sialidase activity spot tests. YS participated in sequencing and in the immunoassays. KR participated in the design of the study. JF conceptualized the study and supervised all facets of the research. All authors read and approved the final manuscript.

\section{Competing interests}

The authors declare that they have no competing interests.

Received: 30 August 2010 Accepted: 2 November 2010 Published: 11 January 2011

\section{References}

1. Nagano N, Isomine S, Kato H, Sasaki Y, Takahashi M, Sakaida K, Nagano Y, Arakawa Y: Human fulminant gas gangrene caused by Clostridium chauvoei. J Clin Microbiol 2008, 46:1545-1547. 
2. Sathish S, Swaminathan K: Molecular characterization of the diversity of Clostridium chauvoei isolates collected from two bovine slaughterhouses: Analysis of cross-contamination. Anaerobe 2008, 14:190-199.

3. Songer JG: Clostridial diseases of small ruminants. Vet Res 1998, 29:219-232.

4. Hatheway CL: Toxigenic clostridia. Clin Microbiol Rev 1990, 3:66-98.

5. Useh NM, Nok AJ, Esievo KA: Pathogenesis and pathology of blackleg in ruminants: the role of toxins and neuraminidase. A short review. Vet $Q$ 2003, 25:155-159.

6. Sasaki Y, Kojima A, Aoki H, Ogikubo Y, Takikawa N, Tamura Y: Phylogenetic analysis and PCR detection of Clostridium chauvoei, Clostridium haemolyticum, Clostridium novyi types A and B, and Clostridium septicum based on the flagellin gene. Vet Microbiol 2002, 86:257-267.

7. Sasaki Y, Yamamoto K, Kojima A, Norimatsu M, Tamura Y: Rapid identification and differentiation of pathogenic clostridia in gas gangrene by polymerase chain reaction based on the 16S-23S rDNA spacer region. Res Vet Sci 2000, 69:289-294.

8. Boraston AB, Ficko-Blean E, Healey M: Carbohydrate recognition by a large sialidase toxin from Clostridium perfringens. Biochemistry 2007, 46:11352-11360.

9. X $\mathrm{X}$ G, Li X, Andrew PW, Taylor GL: Structure of the catalytic domain of Streptococcus pneumoniae sialidase NanA. Acta Crystallogr Sect F Struct Biol Cryst Commun 2008, 64:772-775.

10. Newstead S, Chien CH, Taylor M, Taylor G: Crystallization and atomic resolution X-ray diffraction of the catalytic domain of the large sialidase, nanl, from Clostridium perfringens. Acta Crystallogr D Biol Crystallogr 2004, 60:2063-2066.

11. Watts AG, Oppezzo P, Withers SG, Alzari PM, Buschiazzo A: Structural and kinetic analysis of two covalent sialosyl-enzyme intermediates on Trypanosoma rangeli sialidase. J Biol Chem 2006, 281:4149-4155.

12. Roggentin P, Rothe B, Kaper JB, Galen J, Lawrisuk L, Vimr ER, Schauer R: Conserved sequences in bacterial and viral sialidases. Glycoconj J 1989, 6:349-353.

13. Vimr ER, Kalivoda KA, Deszo EL, Steenbergen SM: Diversity of microbial sialic acid metabolism. Microbiol Mol Biol Rev 2004, 68:132-153.

14. Corfield T: Bacterial sialidases-roles in pathogenicity and nutrition. Glycobiology 1992, 2:509-521.

15. Colman PM: Influenza virus neuraminidase: structure, antibodies, and inhibitors. Protein Sci 1994, 3:1687-1696.

16. Manco $S$, Hernon F, Yesilkaya H, Paton JC, Andrew PW, Kadioglu A: Pneumococcal neuraminidases $A$ and $B$ both have essential roles during infection of the respiratory tract and sepsis. Infect Immun 2006, 74:4014-4020

17. Tong HH, Li D, Chen S, Long JP, DeMaria TF: Immunization with recombinant Streptococcus pneumoniae neuraminidase NanA protects chinchillas against nasopharyngeal colonization. Infect Immun 2005, 73:7775-7778.

18. Soong G, Muir A, Gomez MI, Waks J, Reddy B, Planet P, Singh PK, Kaneko Y, Wolfgang MC, Hsiao YS, Tong L, Prince A: Bacterial neuraminidase facilitates mucosal infection by participating in biofilm production. I Clin Invest 2006, 116:2297-2305.

19. Mally M, Shin H, Paroz C, Landmann R, Cornelis GR: Capnocytophaga canimorsus: a human pathogen feeding at the surface of epithelial cells and phagocytes. PLoS Pathog 2008, 4:e1000164.

20. Tierney DM, Strauss LP, Sanchez JL: Capnocytophaga canimorsus mycotic abdominal aortic aneurysm: why the mailman is afraid of dogs. $J$ Clin Microbiol 2006, 44:649-651.

21. Müller HE, Schallehn G: Occurrence of neuraminidase and acylneuraminat-lyase in Clostridium chauvoei (Clostridium feseri). Zentralbl Bakteriol Orig A 1972, 222:64-69, (in German).

22. Heuermann $D$, Roggentin $P$, Kleineidam RG, Schauer R: Purification and characterization of a sialidase from Clostridium chauvoei NC08596. Glycoconjugate J 1991, 8:95-101.

23. Useh NM, Ajanusi JO, Esievo KA, Nok AJ: Characterization of a sialidase (neuraminidase) isolated from Clostridium chauvoei (Jakari strain). Cell Biochem Funct 2006, 24:347-352.

24. Pitcher DG, Saunders NA, Owen RJ: Rapid extraction of bacterial genomic DNA with guanidium thiocyanate. Lett Appl Microbiol 1989, 8:151-156.

25. Ausubel FM, Brent R, Kingston RE, Moore DD, Seidman JG, Smith JA, Struhl K: Current protocols in molecular biology. New York: John Wiley \& Sons, Inc; 1999.
26. BLAST: Basic local alignment search tool. [http://blast.ncbi.nlm.nih.gov/ Blast.cgi].

27. ExPASy - ProtParam tool. [http://www.expasy.ch/tools/protparam.html].

28. Motif Scan. [http://hits.isb-sib.ch/cgi-bin/motif_scan].

29. InterProScan - Signature recognition search against the integrated resource of protein domains and functional sites. [http://www.ebi.ac.uk/ Tools/InterProscan/]

30. SignalP 3.0 Server. [http://www.cbs.dtu.dk/services/SignalP/].

31. HMMTOP: Prediction of transmembrane helices and topology of proteins - Version 2.0. [http://www.enzim.hu/hmmtop/index.html].

32. Phobius - A combined transmembrane topology and signal peptide predictor. [http://phobius.sbc.su.se/]

33. Align Sequences using ClustalW2 | EBI. [http://www.ebi.ac.uk/Tools/ clustalw2/index.html].

34. Rothe B, Rothe B, Roggentin P, Schauer R: The sialidase gene from Clostridium septicum: cloning, sequencing, expression in Escherichia coli and identification of conserved sequences in sialidases and other proteins. Mol Gen Genet 1991, 226:190-197.

35. Schaller A, Kuhn R, Kuhnert P, Nicolet J, Anderson TJ, Maclnnes Jl, Segers RPAM, Frey J: Characterization of apxIVA, a new RTX determinant of Actinobacillus pleuropneumoniae. Microbiology 1999, 145:2105-2116.

36. Studier FW, Rosenberg AH, Dunn JJ, Dubendorff JW: Use of T7 RNA polymerase to direct expression of cloned genes. Methods Enzymol 1990, 185:60-89.

37. Vilei EM, Abdo E-M, Nicolet J, Botelho A, Gonçalves R, Frey J: Genomic and antigenic differences between the European and African/Australian clusters of Mycoplasma mycoides subsp. mycoides SC. Microbiology 2000, 146:477-486.

38. Laemmli UK: Cleavage of structural proteins during the assembly of the head of bacteriophage T4. Nature 1970, 227:680-685.

39. Lewin A, Mayer M, Chusainow J, Jacob D, Appel B: Viral promoters can initiate expression of toxin genes introduced into Escherichia coli. BMC Biotechnol 2005, 5:19.

40. Moncla BJ, Braham P: Detection of sialidase (neuraminidase) activity in Actinomyces species by using 2'-(4-methylumbelliferyl)alpha-D-Nacetylneuraminic acid in a filter paper spot test. J Clin Microbiol 1989, 27:182-184.

41. Munzert $E$, Müthing J, Büntemeyer $H$, Lehmann J: Sialidase activity in culture fluid of Chinese hamster ovary cells during batch culture and its effect on recombinant human antithrombin III integrity. Biotechnol Prog 1996, 12:559-563.

42. Roggentin P, Krug G, Schauer R, Brasch J: Lack of sialidase activity in Candida albicans and Candida glabrata. Mycoses 1999, 42:33-36.

43. Berry AM, Lock RA, Paton JC: Cloning and characterization of nanB, a second Streptococcus pneumoniae neuraminidase gene, and purification of the NanB enzyme from recombinant Escherichia coli. J Bacteriol 1996, 178:4854-4860

44. Lock RA, Paton JC, Hansman D: Purification and immunological characterization of neuraminidase produced by Streptococcus pneumoniae. Microb Pathog 1988, 4:33-43.

45. Grobe K, Sartori B, Traving C, Schauer R, Roggentin P: Enzymatic and molecular properties of the Clostridium tertium sialidase. J Biochem 1998, 124:1101-1110.

46. Zenz Kl, Roggentin P, Schauer R: Isolation and properties of the natural and the recombinant sialidase from Clostridium septicum NC 0054714. Glycoconj J 1993, 10:50-56.

47. Kuhnert P, Capaul S, Nicolet J, Frey J: Phylogenetic positions of Clostridium chauvoei and Clostridium septicum based on 16S rRNA gene sequences. Int J Syst Bacteriol 1996, 46:1174-1176.

48. Moussa RS: Antigenic formulae for Clostridium septicum and Clostridium chauvoei. J Pathol Bact 1959, 77:341-350.

49. Nakatsuji T, Liu YT, Huang CP, Gallo RL, Huang CM: Vaccination targeting a surface sialidase of $P$. acnes: implication for new treatment of acne vulgaris. PLOS ONE 2008, 3:e1551.

50. Long JP, Tong HH, DeMaria TF: Immunization with native or recombinant Streptococcus pneumoniae neuraminidase affords protection in the chinchilla otitis media model. Infect Immun 2004, 72:4309-4313.

doi:10.1186/1297-9716-42-2

Cite this article as: Vilei et al: Genetic and functional characterization of the NanA sialidase from Clostridium chauvoei. Veterinary Research 2011 42:2. 